\title{
Facial Oedema Is Not Always Angioedema: A Case of Spontaneous Pneumomediastinum with Subcutaneous Emphysema during COPD Exacerbation
}

\author{
Sarah Damanti ${ }^{1}$, Francesca Rosini ${ }^{1}$, Daniela Mari ${ }^{2,3}$, Maura Marcucci $^{2,3}$ \\ ${ }^{1}$ School of Specialization in Geriatrics and Gerontology, Università degli Studi di Milano, Milan, Italy \\ ${ }^{2}$ Geriatric Unit, Fondazione IRCCS Ca' Granda Ospedale Maggiore Policlinico, Milan, Italy \\ ${ }^{3}$ Department of Clinical Sciences \& Community Health, University of Milan, Milan, Italy
}

Received: 02/09/2015

Accepted: 30/09/2015

Published: 28/10/2015

How to cite this article: Damanti S, Rosini F, Mari D, Marcucci M. Facial oedema is not always angioedema: a case of spontaneous pneumomediastinum with subcutaneous emphysema during COPD exacerbation. EJCRIM 2015;2:doi:10.12890/2015_000281

Conflicts of Interests: The authors declare that there are no competing interests.

Patient's Consent: The authors declare they obtained the consent for publication from the patient's daughter.

This article is licensed under a Commons Attribution Non-Commercial 4.0 License

\section{ABSTRACT}

We report a case of acute facial oedema in an elderly hospitalized patient which was initially misdiagnosed as angioedema secondary to antibiotics in a patient with an allergic diathesis. We describe the differential aetiologies and then the true cause of the oedema, which was an uncommon complication of a very common condition in the elderly: a pneumomediastinum with subcutaneous emphysema probably due to rupture of an emphysematous lung bulla during chronic obstructive pulmonary disease (COPD) exacerbation. Lastly, we focus on the therapeutic procedures instituted for the treatment of the pneumomediastinum.

\section{LEARNING POINTS}

- It is difficult to collect medical history and drug allergy information from elderly hospitalized patients because of cognitive impairment or confusion.

- When reviewing the differential diagnosis, it is very important to avoid fixation errors as they can seriously affect the patient's prognosis.

- Unusual complications of extremely common chronic diseases among older people must also be considered during clinical reasoning.

\section{KEYWORDS}

Pneumomediastinum; COPD exacerbation; angioedema.

\section{CASE REPORT}

Two days after admission to a medical ward for chronic obstructive pulmonary disease (COPD) exacerbation, a 90-year-old woman affected by cognitive decline developed acute facial oedema, mainly localized in the periorbital and palpebral areas (Fig. 1).

At admission she had not been able to reliably describe her medical history so she had been treated with levofloxacin, which was withdrawn $24 \mathrm{~h}$ later when her daughter reported a possible allergy to fluoroquinolones. 
In this scenario, we first hypothesized that the facial oedema, despite the absence of itching and urticaria, was the manifestation of an allergic reaction. However, symptoms did not improve when glucocorticoids were administered intravenously. Indeed, the oedema rapidly worsened until the patient was unable to open her eyes. The patient was eupnoic, haemodynamically stable and was not complaining of chest pain. While examining the patient and considering alternative diagnoses, we touched her neck and felt a 'crushed snow' sensation. A facial, neck and chest CT scan then revealed a pneumomediastinum associated with severe subcutaneous emphysema, which was probably secondary to rupture of an emphysematous lung bulla during a coughing paroxysm ${ }^{[1]}$.

In light of the absence of mediastinal organ injury, we adopted a conservative approach ${ }^{[2,3]}$. We administered antibiotics and oxygen, and prescribed rest, antitussives and laxatives in order to prevent any movement that could increase the intrathoracic pressure. Positioning needles in the subcutis was also effective to facilitate percutaneous air drainage (Fig. 2).

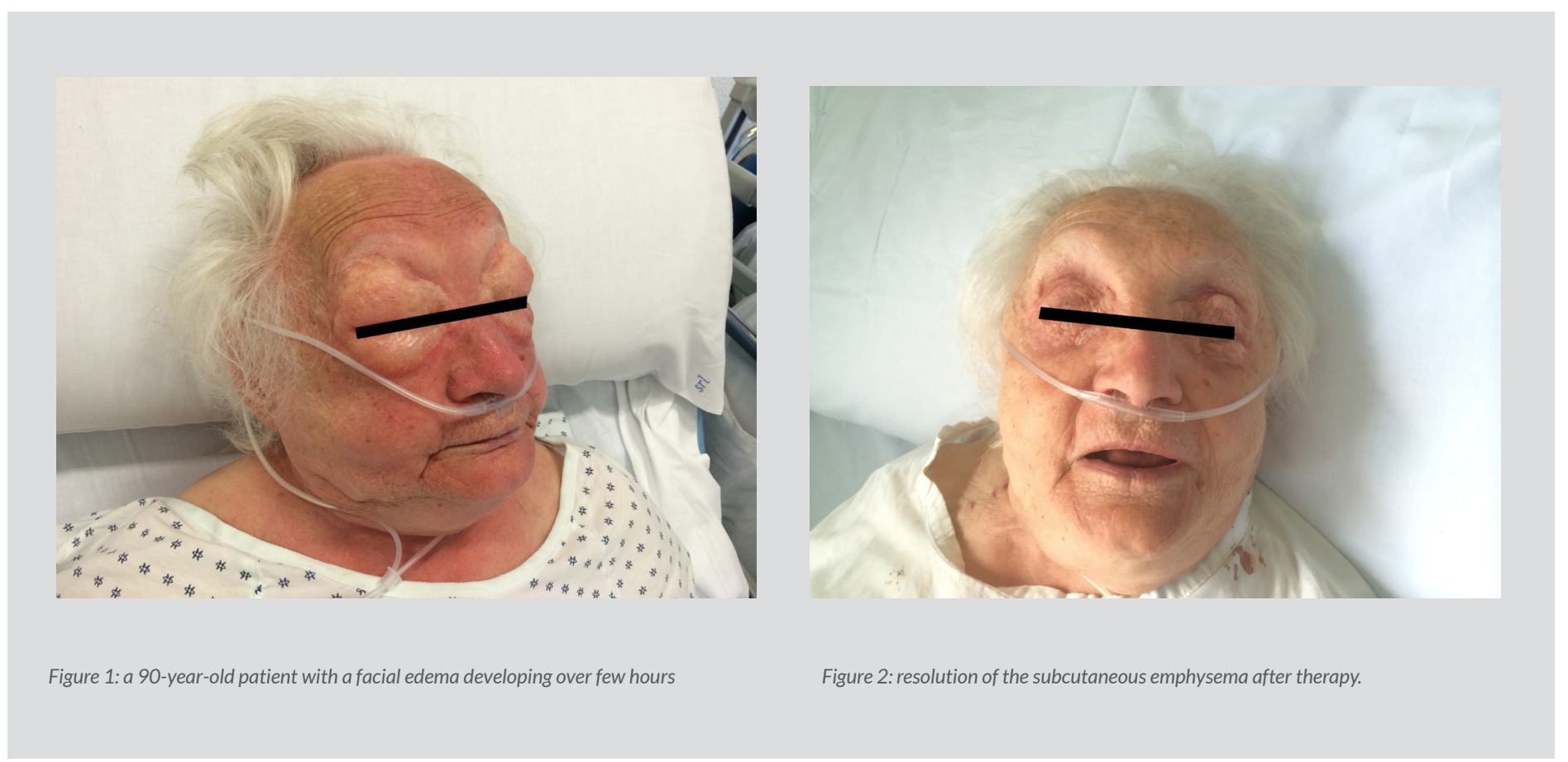

\section{DISCUSSION}

Spontaneous pneumomediastinum is uncommon ${ }^{[2]}$. It might be caused by rupture of emphysematous bullae causing leakage of air through the pulmonary interstitium towards the mediastinum ${ }^{[4]}$. A severe cough, such as associated with COPD exacerbation, can precipitate such a rupture.

When pneumomediastinum is associated with facial subcutaneous emphysema in patients with a suspected allergic diathesis, it must be properly differentiated from angioedema due to anaphylaxis. Both are potentially life-threatening conditions, especially in elderly patients with limited pulmonary reserve, and must be managed promptly. Our patient lacked some manifestations related to mast cell mediator release (urticaria, flushing, pruritus, bronchospasm and hypotension), but angioedema can be the only sign of anaphylaxis. Additional alternative causes of acute/subacute facial oedema to consider include: (a) erysipelas, but the oedema typically presents as a welldemarcated erythematous and warm plaque; (b) a large local reaction to an insect bite, which we would expect to respond to steroids; (c) zoster, but without the usual pain, vesicular skin lesions and unilateral presentation; and (d) a superior vena cava syndrome due to a massive thoracic vein thrombosis or to a rapidly increasing tumour mass. Therefore, the vital signs of a patient with facial oedema should be monitored carefully since it can be the manifestation of a condition that can severely interfere with haemodynamic and respiratory function. This clinical case highlights a few important aspects which should be taken into account. First, it is not easy to prescribe a new drug in elderly hospitalized patients who are unable to provide reliable information about their medical history and drug allergies because of cognitive impairment or confusion. Therefore, it is fundamental to confer with the patient's caregivers. Second, clinical reasoning can sometimes be influenced by 'fixation errors'. In our case we swiftly administered steroids because we relied on the patient's history of allergy without actually examining the patient. Finally, in the differential diagnosis, it is important to also consider unusual complications of extremely common chronic diseases when dealing with multi-morbid elderly patients with complex clinical scenarios. 


\section{REFERENCES}

1. Bakhos CT, Pupovac SS, Ata A, Fantauzzi JP, Fabian T. Spontaneous pneumomediastinum: an extensive workup is not required. J Am Coll Surg 2014;219:713-717.

2. Banki F, Estrera AL, Harrison RG, Miller CC 3rd, Leake SS, Mitchell KG, et al. Pneumomediastinum: etiology and a guide to diagnosis and treatment. Am J Surg 2013;206:10011006.

3. Mansella G, Bingisser R, Nickel CH. Pneumomediastinum in blunt chest trauma: a case report and review of the literature. Case Rep Emerg Med 2014;2014:685381.

4. Murayama S, Gibo S. Spontaneous pneumomediastinum and Macklin effect: overview and appearance on computed tomography. World J Radiol 2014;6:850-854. 